\title{
Lack of high-quality studies comparing the effectiveness, and cost-effectiveness, of dental auxiliaries and dentists in performing dental care
}

\author{
Abstracted from \\ Dyer TA, Brocklehurst P, Glenny AM, Davies L, Tickle M, Issac A, Robinson PG. \\ Dental auxiliaries for dental care traditionally provided by dentists. \\ Cochrane Database Syst Rev 2014; 8: Art. No. CD010076. DOI: 10.1002/14651858.CD010076.pub2. \\ Address for correspondence: Tom A Dyer, School of Clinical Dentistry, University of Sheffield, \\ Claremont Crescent, Sheffield, S10 2TA, UK. Email: t.dyer@sheffield.ac.uk
}

\section{Question: Is using dental auxiliaries to provide care traditionally delivered by dentists effective?}

Data sources Cochrane Effective Practice and Organisation of Care (EPOC) Group's Specialised Register; Cochrane Oral Health Group's Specialised Register; the Cochrane Central Register of Controlled Trials Medline; Embase; CINAHL; Cochrane Database of Systematic Reviews; Database of Abstracts of Reviews of Effectiveness; five other databases and two trial registries. A number of dental journals were handsearched and a grey literature search preformed.

Study selection Randomised controlled trials (RCTs), non-randomised controlled trials (NRCTs), controlled before and after studies (CBAs) and interrupted time series (ITSs) were considered. Selection was conducted independently by two reviewers.

Data extraction and synthesis Three reviewers extracted data and assessed risk of bias. Meta-analysis was not possible so a narrative summary was presented.

Results Five studies (one cluster RCT, three RCTs and one NRCT) were included. All the studies were at high risk of bias and the overall quality of evidence was very low. The majority of the studies were more than 20 years old.

Four studies evaluated sealant placement; three found no evidence of a difference in retention rates of those placed by dental auxiliaries and dentists over a range of follow-up periods (six to 24 months). One study found that sealants placed by a dental auxiliary had lower retention rates than ones placed by a dentist after 48 months ( $9.0 \%$ with auxiliary versus $29.1 \%$ with dentist); but the net reduction in the number of teeth exhibiting caries was lower for teeth treated by the dental auxiliary than the dentist (three with auxiliary versus 60 with dentist, $P$ value $<0.001)$. One study showed no evidence of a difference in dental decay after treatment with fissure sealants between groups. One study comparing the effectiveness of dental auxiliaries and dentists performing ART reported no difference in survival rates of the restorations (fillings) after 12 months.

Conclusions We only identified five studies for inclusion in this review, all of which were at high risk of bias, and four were published more than 20 years ago, highlighting the paucity of high-quality evaluations of the relative effectiveness, cost-effectiveness and safety of dental auxiliaries compared with dentists in performing clinical tasks. No firm conclusions could be drawn from the present review about the relative effectiveness of dental auxiliaries and dentists.

\section{Commentary}

As expected from a properly guided Cochrane Review this review follows generally accepted guidelines to conduct and report a systematic review. It has to be noted that this is an update from a review first published in 2010. An update after three years is well received, even though the conclusions remained unchanged. The stated objectives are clinically important when properly framed and the considered outcomes are important for both the clinician (level and alignment, root resorption) and the patient (pain intensity). One relatively significant deficiency of this review is not having included electronic databases that include articles published from Brazil, China and Turkey in their original languages. These countries have consistently published clinical trials in the last decade. Maybe some additional RCTs could have been missed.

Regarding the quality of included studies, only RCTs with a fully bonded dental arch were included. Although the number of included RCTs is respectable, the total number of included participants is not overall impressive. For the specific review questions the unit of analysis is the participant not the number of included teeth. When the methodological quality of the included RCTs is considered it becomes clear that the results need to be considered very cautiously due to the high risk of bias among all the studies.

None of the included studies did quantify or qualify the amount of root resoption produced. So there is no answer to that question. In regards to any specific type of wire marketed as an initial orthodontic wire being superior for level and alignment, this systematic review failed to find any consistent evidence. The same applies for pain intensity.

In summary, the available evidence failed to justify consistently the selection of any specific initial level and alignment orthodontic wire as a superior option. We have to keep in mind that lack of evidence is not automatically proof of no difference.

Derek Richards

Centre for Evidence-based Dentistry, Dental Health Services Unit, Dundee Dental Hospital and School, University of Dundee, Scotland

1. Sibbald B, McBride A, Birch S. Labour substitution and efficiency in health care delivery: general principles and key messages. Centre for Workforce intelligence.London 2011. (Downloaded from http://www.cfwi.org.uk/publications/labour-substitution-andefficiency-in-healthcare-delivery-general-principles-and-key-messages)

2. Wright JT, Graham F, Hayes C, et al. A systematic review of oral health outcomes produced by dental teams incorporating midlevel providers. J Am Dent Assoc 2013; 144: 75-91.

3. Galloway J, Gorham J, Lambert M, et al. The Professionals' Complementary to Dentistry: A Systematic Review and Synthesis. London: University College London, Eastman Dental Hospital, Dental Team Studies Unit, 2002.

Evidence-Based Dentistry (2014) 15, 80. doi:10.1038/sj.ebd.6401043 